Cómo citar este trabajo: Carrillo-Hidalgo, I., Pulido-Fernández, J. I., \& Mudarra-Fernández, A. B. (2019). Caracterización del turista en Ciudades Patrimonio de la Humanidad: el caso de Úbeda y Baeza. Boletín de la Asociación de Geógrafos Españoles, 81, 2746, 1-25. http://dx.doi.org/10.21138/bage.2746

\title{
Caracterización del turista en Ciudades Patrimonio de la Humanidad: el caso de Úbeda y Baeza
}

Characterization of the tourist in World Heritage Cities: the case of úbeda and Baeza

\author{
Isabel Carrillo-Hidalgo \\ ihidalgo@ujaen.es \\ Juan Ignacio Pulido-Fernández \\ jipulido@ujaen.es \\ Ana Belén Mudarra-Fernández \\ anab.mudarra@gmail.com \\ Laboratorio de Análisis e Innovación Turística \\ Universidad de Jaén (España)
}

\section{Resumen}

Úbeda y Baeza fueron declaradas Ciudades Patrimonio de la Humanidad en 2003. Desde entonces, el turismo en estas ciudades se ha convertido en una actividad clave para su economía y se ha incrementado el número de llegadas en más de un cincuenta por ciento. La gestión de cualquier Ciudad Patrimonio de la Humanidad requiere conocer en profundidad al visitante desde diferentes perspectivas: variables sociodemográficas y psicológicas del turista, características del viaje, actividades llevadas a cabo en el destino, la satisfacción con los recursos de este, así como del gasto realizado por el turista durante el viaje y el presupuesto del que parte. En concreto, para el caso de Úbeda y Baeza, se ha podido comprobar que el perfil del turista que las visita coincide a grandes rasgos con el del que acude a otras Ciudades Patrimonio de la Humanidad, establecido en 
la literatura científica, diferenciándose principalmente en variables más específicas relacionadas con el destino y la propia personalidad del turista. Los resultados alcanzados aportan una información de gran utilidad para gestores y policymakers de las Ciudades Patrimonio de la Humanidad, que podrán adaptar su oferta turística al turista que las visita.

Palabras clave: Ciudades Patrimonio de la Humanidad; turismo cultural; perfil del turista; gasto turístico.

\begin{abstract}
Úbeda and Baeza were declared World Heritage Cities in 2003. Since then, tourism in these cities has become a key activity for their economy and the number of arrivals has increased by more than fifty percent. The management of any World Heritage City requires an in-depth knowledge of the tourist who visits it from different perspectives: socio-demographic and psychological variables of the tourist, characteristics of the trip, activities carried out in the destination, satisfaction with the resources of this, as well as the tourist expenditure during the trip and the budget from which he departs. Specifically, in the case of Ubeda and Baeza, it has been possible to verify that the profile of the tourist who visits them coincides roughly with the profile of the tourist that goes to other World Heritage Cities, established in the scientific literature, differing mainly in more specific variables related to the destination and the tourist's own personality. The results achieved provide very useful information for managers and policymakers of the World Heritage Cities, who can adapt their tourist offer to the tourist who visits them.
\end{abstract}

Key words: traits of tourist; World Heritage Cities; cultural tourism; tourist profile; tourist expenditure.

\title{
1 Introducción
}

El turismo es la actividad responsable de movimientos de millones de personas entre diferentes lugares del mundo, llegando a aumentar, desde mediados del siglo pasado, de 25 millones de llegadas internacionales hasta los 1.323 millones en 2017 (UNWTO, 2018).

Los atractivos culturales y/o naturales de los destinos conducen a una mayor demanda turística, especialmente los reconocidos oficialmente, es decir, inscritos en la Lista del Patrimonio de la Humanidad por la UNESCO (Su \& Lin, 2014). De manera que, desde hace décadas, es creciente la incorporación de las ciudades patrimoniales a los destinos turísticos.

Por su parte, el turismo cultural y patrimonial, además de generar los beneficios propios del turismo en general, como la mejora de la competitividad, la creación de empleo, el crecimiento económico, la ralentización de la despoblación rural, etc., genera ingresos que permiten la conservación del patrimonio y cultiva un sentimiento de orgullo y autoestima de la población de los destinos 
(UNWTO, 2015). Aunque, si los recursos patrimoniales y culturales no son gestionados como se debiera, los beneficios asociados no están siempre garantizados (Pulido-Fernández \& Ruiz-Lanuza, 2017).

Los patrones de visita de los turistas están relacionados con las características personales de los participantes, sus opiniones y los significados que estos asocian a los diferentes espacios de los destinos (Poria et al., 2003) y, en cuanto al turismo cultural y patrimonial, éste debe basarse en el vínculo existente entre el individuo y el espacio, es decir, las percepciones turísticas de un sitio en relación con su patrimonio (Poria et al., 2006).

Dado el crecimiento exponencial del turismo en general, y del cultural en particular, surge la necesidad de analizar la demanda y flujos turísticos, la motivación de los visitantes, el perfil de los turistas, el comportamiento de estos, la planificación de los viajes, empleo de las TIC, la fidelidad de los turistas etc. Líneas de investigación consideradas como prioritarias por Pulido-Fernández y Ruiz-Lanuza (2017). De manera que se logre caracterizar al turista que visita diferentes destinos, mejorando así el conjunto de conocimientos en este campo para los gestores turísticos.

En 2018, se cumplen quince años de la inscripción de los conjuntos monumentales renacentistas de Úbeda y Baeza como Ciudades Patrimonio de la Humanidad -en adelante, CPH-, debido a la cantidad de recursos culturales y patrimoniales de carácter renacentista que atesoran estas ciudades. La inclusión de Úbeda y Baeza en el grupo de CPH ha duplicado el número de visitantes que han recibido desde 2003 (Cárdenas-García et al., 2014), llegando a aumentar en un 50 \% el número de pernoctaciones desde 2012 (Instituto de Estadística y Cartografía de Andalucía, 2018).

El análisis del perfil del turista que visita Úbeda y Baeza permite caracterizar el tipo de visitante que se siente atraído por estas CPH en función de:

- Las variables sociodemográficas y psicológicas del turista.

- Las características del viaje que realiza.

- Las actividades realizadas en el destino.

- La satisfacción con los recursos de este.

- El gasto realizado por el turista durante el viaje.

- El presupuesto del que parte.

Para ello, se ha llevado a cabo una profunda revisión bibliográfica sobre los trabajos realizados en materia de caracterización del turista en $\mathrm{CPH}$, que queda reflejada en el marco teórico, para, a continuación, realizar un cuestionario a los turistas de estas $\mathrm{CPH}$, el cual permite perfilar, en el apartado de resultados y discusión, el tipo de turista que las visita y cómo se comporta, que posibilita comparar los resultados obtenidos en este análisis empírico con anteriores investigaciones en este sentido en las conclusiones finales. 
Se parte de la hipótesis de que el perfil del turista que visita Úbeda y Baeza coincide en gran parte con la caracterización establecida por la literatura científica sobre el turista que visita otras $\mathrm{CPH}$, realizada en el apartado siguiente, salvando ciertas diferencias relacionadas con las características del viaje y del destino, y de la propia personalidad del turista.

Los resultados obtenidos son de gran interés para la toma de decisiones en la gestión del turismo en las ciudades de Úbeda y Baeza y en otras $\mathrm{CPH}$.

\section{Marco teórico y revisión bibliográfica}

Comprender qué es el turismo cultural ha dado lugar a diferentes definiciones y modificaciones de éstas. Fue la UNESCO la que estableció una primera definición formal, describiendo el turismo cultural como la forma de turismo cuyo objeto es descubrir y conocer monumentos y lugares histórico-artísticos, ejerciendo un efecto realmente positivo sobre éstos, ya que contribuye a su mantenimiento y protección (ICOMOS, 1976), siendo uno de las principales prioridades y preocupaciones de la UNESCO (2014). Es la Organización Mundial del Turismo la que aporta una definición comúnmente aceptada, estableciendo que el turismo cultural es:

el desplazamiento de personas a atracciones culturales en destinos distintos a su lugar de residencia, con la intención de reunir nueva información y experiencias para satisfacer sus necesidades culturales (UNWTO, 2005, p. 6)

No obstante, el turismo cultural es un concepto en permanente evolución, ya que engloba, por un lado, la demanda y las motivaciones y necesidades de ésta. Y, por otro lado, la oferta de recursos contextualizados en el patrimonio monumental y material e inmaterial, es decir, cultural (Morére \& Perelló, 2013).

Esta continua transformación del concepto de turismo cultural queda reflejada en una de las últimas definiciones académicas es la aportada por Richards (2018, p. 13) en la que establece que:

el turismo cultural es un tipo de actividad turística en la que la motivación esencial del visitante es aprender, descubrir, experimentar y consumir las atracciones/productos culturales tangibles e intangibles en un destino turístico.

El turismo cultural como una forma de consumo cultural ha sido un tema particularmente importante para la literatura científica, tratado de comprender la quién consume turismo cultural y, en particular, la variación y la estratificación dentro de ella (Richards, 2018). La demanda turística, normalmente, se divide en función de las características sociodemográficas, geográficas y psicológicas, de manera que resulta fundamental entender el tipo de turistas, su origen y cómo y por qué se comportan de determinadas maneras, para poder adaptar la oferta turística a sus expectativas y necesidades. De este modo, el análisis del perfil del turista es fundamental para la gestión de un destino (Poria et al., 2001). 
Respecto al turismo cultural, es cierto que los análisis del perfil del turista atraído por estos destinos se han realizado principalmente desde la perspectiva socio-demográfica, llegando a enmarcarlo dentro del grupo de turistas maduros, con posibilidades económicas medio-altas, y con capital cultural $^{1}$ (Chhabra, Healy \& Sills, 2003; Craik, 1997; Kim et al., 2007; Munt, 1994; Richards, 1996; Silverberg, 1995; Zeppel \& Hall, 1991). Un estudio realizado por Pulido-Fernández y Sánchez-Rivero (2010) concluye que la edad y, aunque en menor medida, el poder adquisitivo del turista influyen de manera directa y positiva en la predisposición a realizar turismo cultural.

Sin embrago, algunos autores (Kim et al., 2007) afirman que esta caracterización del turista es muy simplista, que no se corresponde con la oferta diversa de turismo cultural y que no se adapta a las nuevas tendencias, ya que no se trata de un turismo homogéneo y monolítico (Pulido-Fernández \& Sánchez-Rivero, 2010).

Poria et al. (2001, p. 1048) argumentan que comprender las motivaciones y percepciones:

es útil para la gestión de destinos [patrimoniales] con respecto a factores como la política de precios, la misión de las atracciones patrimoniales y la comprensión de los perfiles de los visitantes, así como la financiación pública y la gestión sostenible ...

Dicha información sería especialmente útil para las comunidades que intentan promover el turismo patrimonial, ya que podría conducir al desarrollo de productos mejor adaptados a los gustos de los turistas potenciales (Chaabra et al., 2003).

De este modo, la reciente literatura científica (Marrero-Rodríguez \& Abdul-Jalbar, 2012; PulidoFernández et al., 2017) ha identificado diferencias en el perfil del turista cultural en base a la motivación de la visita, la valoración otorgada a los servicios y patrimonio de los destinos culturales, el comportamiento y experiencia durante la visita y los lugares visitados, la satisfacción con la visita, el atractivo por los lugares de interés cultural, la motivación del viaje, la edad y gasto realizado o la fidelidad con el destino.

Así, los estudios del perfil del turista que se vienen realizando desde los años 90 del pasado siglo han evolucionado, pasando de analizar cómo es el turista que visita los destinos a cómo piensa y se comporta, ya que el propio turista ha evolucionado de una antigua cultura consumidora como parte de una experiencia general de vacaciones, a viajar buscando participar en algún aspecto de la cultura del destino (Richards, 2018).

1 Según Bordieu (1986) el capital cultural es la acumulación de cultura propia que se da en las personas en tres estados diferentes:

- Incorporado: intrínsecos en la propia persona, dando lujar al hábito, que incluye el comportamiento corporal y el habla como marcadores de distinción.

- Objetivado: referidos a los bienes culturales propiedad de la persona.

- Institucionalizado: la educación formal recibida. 
Son diversos los rasgos y comportamientos comunes de los turistas que visitan las CPH. La literatura científica, en su mayoría a partir de estudios de caso (Pulido-Fernández \& Ruiz-Lanuza; 2017), a través de cuestionarios y entrevistas estructuradas o semiestructuradas (Balcar \& Pearce, 1996; Chaabra et al., 2003; Correia et al., 2010; Gregori \& Jarauta, 2015; Junta de Andalucía, 2010; Lara \& López, 2004; Lynch et al., 2010; Marrero-Rodríguez \& Abdul-Jalbar, 2012; MoralesFernández \& Lanquar, 2014; Prada-Trigo et al., 2015; Richards, 2002; Van der Borg et al., 1996), ha perfilado al turista que visita las CPH como hombres y mujeres adultos, mayores de 30 años (llegando algunos a relacionar la variable edad con el envejecimiento de la población), con niveles educativos altos y de capital cultural, así como ocupados, de renta media-alta, en su mayoría nacionales (aunque el estudio realizado por Morales-Fernández y Lanquar (2014) señala que predominan los de nacionalidad extranjera) y que gastan más que en otro tipo de destinos. El destino lo conocen en su mayoría por recomendaciones de familia y amigos.

Respecto a cómo se comportan durante la visita, la literatura científica destaca que la mayoría son turistas satisfechos, que planifican previamente la visita - excepto el estudio realizado por Balcar y Pearce (1996) -, viajan acompañados, preferentemente en pareja, por ocio y vacaciones y las principales actividades que realizan son visitas culturales, pasear y recorrer la ciudad y hacer compras.

Por otra parte, el número de pernoctaciones depende de dónde se haya llevado a cabo el estudio. En CPH de tamaño medio, pernoctan entre una y tres noches en hoteles, normalmente en fin de semana (Balcar \& Pearce, 1996; Marrero-Rodríguez \& Abdul-Jalbar, 2012; Prada-Trigo et al., 2015). Sin embargo, cuando se trata de ciudades de gran tamaño y capitales de países, el número de pernoctaciones es bastante superior, próximo a 7 días (Correia et al., 2010). Igual ocurre al diferenciar si se trata de un turista nuevo (Gregori \& Jarauta, 2015; Prada-Trigo et al., 2015) o fiel (Correia et al., 2010).

Además, algunos estudios llevados a cabo en CPH de carácter religioso analizan el perfil religioso del turista para entender el comportamiento de la demanda (Poria, Butler \& Airey, 2003).

Al fin y al cabo, a la hora de caracterizar al turista de un destino, el principal objetivo de la literatura científica es ser útil a los gestores de destinos turísticos, ya que resulta fundamental conocer el perfil, características, percepción y comportamiento del turista a la hora de viajar, para adaptar la oferta turística a las necesidades y expectativas de los turistas y así ser destinos más competitivos, atrayendo a un mayor número de turistas y de más calidad, teniendo en cuenta que cada tipo de actividad tiene un nivel distinto de gasto asociado (Kruger \& Saayman, 2014). En particular, como destacan Pulido-Fernández et al. (2016), en el caso del turismo cultural y patrimonial, es fundamental generan conocimiento que aporte a los gestores turísticos la información necesaria para crear productos turísticos de alto valor añadido, que favorezcan el impacto económico 
generado por el turismo, ya que existen actividades en este tipo de destinos que determinan el gasto turístico en los destinos analizados.

\section{Metodología}

\subsection{Caso de estudio}

Úbeda y Baeza son dos ciudades situadas en la provincia de Jaén (España) (Figura 1) en las que el turismo, la agricultura y los talleres artesanos son sus principales fuentes de ingresos (CárdenasGarcía et al., 2014). Estas son conocidas por los turistas desde finales del siglo XIX y principios del XX, pero no fue hasta 2003, cuando la UNESCO las declaró Patrimonio de la Humanidad bajo el título de "Dualidad urbana y unidad cultural de Úbeda y Baeza", cuando se fomentó el desarrollo turístico de éstas. En la actualidad, Úbeda y Baeza cuentan con una gran diversidad de recursos que son capaces de satisfacer las necesidades de turistas culturales, debido a su rico patrimonio y a la multitud de festivales, ferias y jornadas que se realizan.

\section{Figura 1. Localización de Úbeda y Baeza (España)}

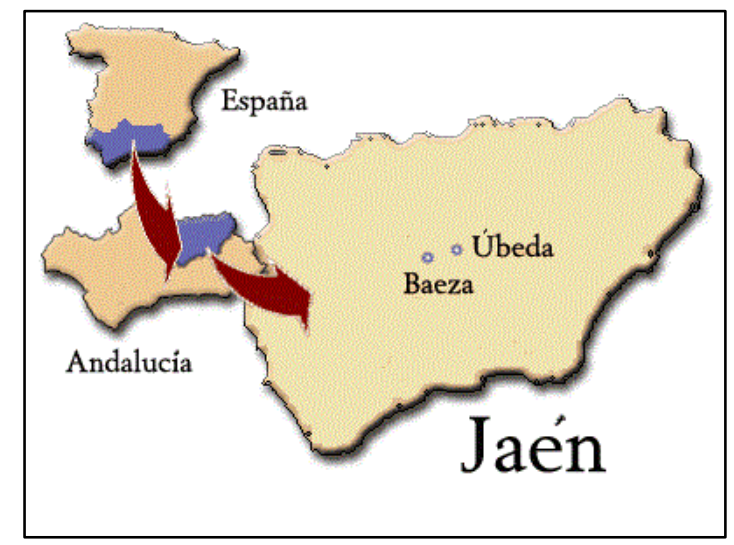

Fuente: López (2014)

La UNESCO se basa en una serie de criterios para considerar un destino como Patrimonio de la Humanidad. A continuación, se detallan los criterios de carácter cultural y de los que Úbeda y Baeza destacan y fueron galardonados con tal mención principalmente por los criterios iii y iv (UNESCO, 2017):

I. Representar una obra maestra del genio creativo humano.

II. Ser testimonio de un importante intercambio de valores humanos a lo largo de un periodo de tiempo o dentro de un área cultural del mundo, en el desarrollo de la arquitectura, tecnología, artes monumentales, urbanismo o diseño paisajístico.

III. Aportar un testimonio único, o al menos excepcional, de una tradición cultural o de una civilización existente o ya desaparecida. 
IV. Ofrecer un ejemplo eminente de un tipo de edificio, conjunto arquitectónico, tecnológico o paisaje, que ilustre una etapa significativa de la historia humana.

V. Ser un ejemplo eminente de una tradición de asentamiento humano, utilización del mar o de la tierra, que sea representativa de una cultura (o culturas), o de la interacción humana con el medio ambiente, especialmente cuando éste se vuelva vulnerable frente al impacto de cambios irreversibles.

VI. Estar directa o tangiblemente asociado con eventos o tradiciones vivas, con ideas o con creencias, con trabajos artísticos y literarios de destacada significación universal.

\section{Figura 2. Plano de Úbeda}

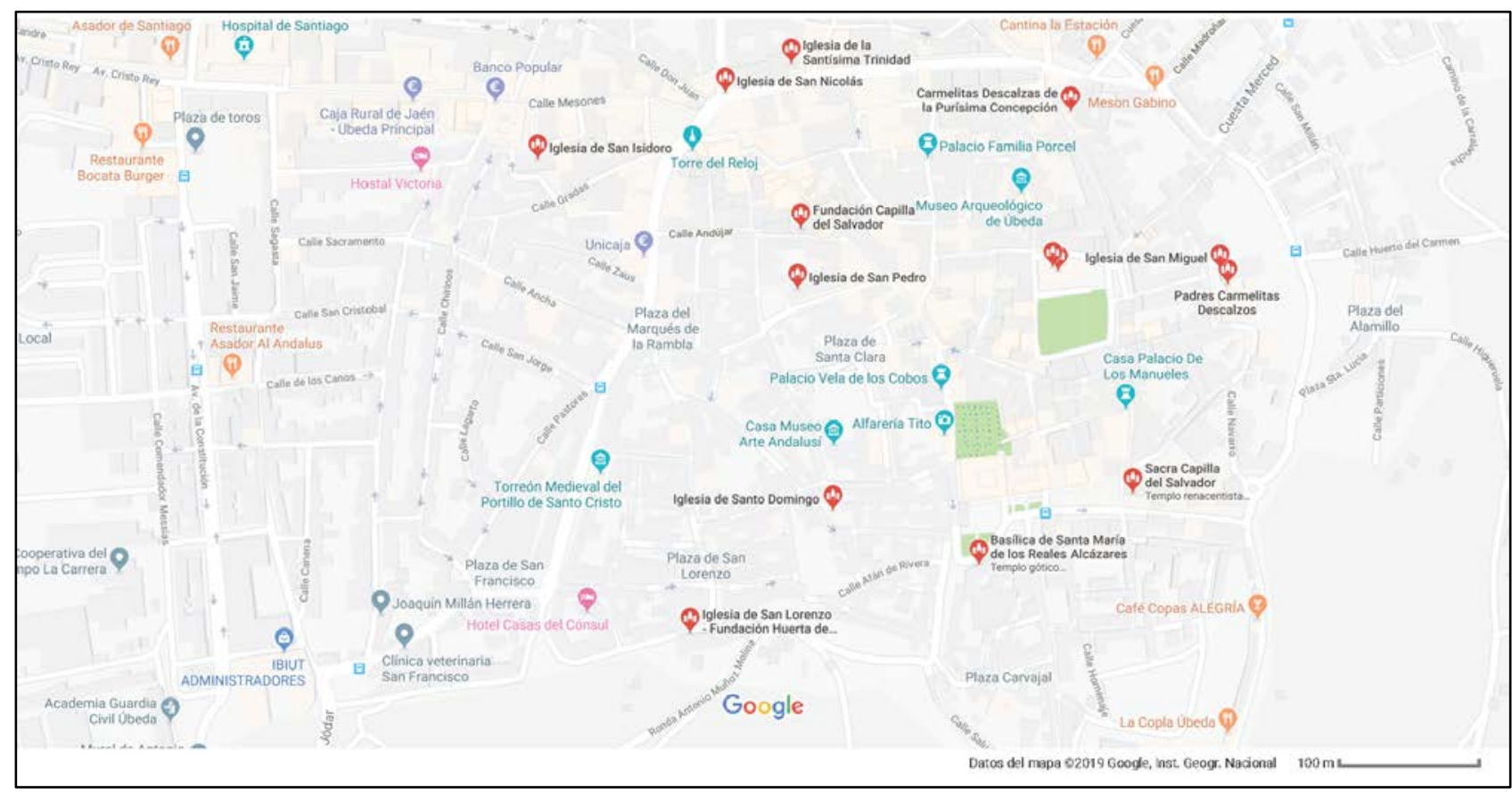

Fuente: Google Maps

El modelo urbanístico y arquitectónico úbeda y Baeza es de origen medieval con influencia de rasgos renacentistas y humanistas procedentes de Italia y ha sido inspiración para numerosas ciudades en el "Nuevo Mundo" (UNESCO, 2009). Las Figuras 2 y 3 recogen la planimetría de los conjuntos monumentales más importantes de ambas ciudades.

Además de los recursos turísticos, las ciudades de Úbeda y Baeza cuentan con un gran número de establecimientos destinados a alojamiento, restauración, infraestructuras de transporte, servicios de información e interpretación públicos y privados, los cuales se revalorizan a través de productos turísticos como itinerarios culturales, gastronomía en relación con la cultura del aceite de oliva, artesanías, eventos, ferias, etc. La gestión del turismo es desempeñada directamente por los propios ayuntamientos. 
Figura 3. Plano de Baeza

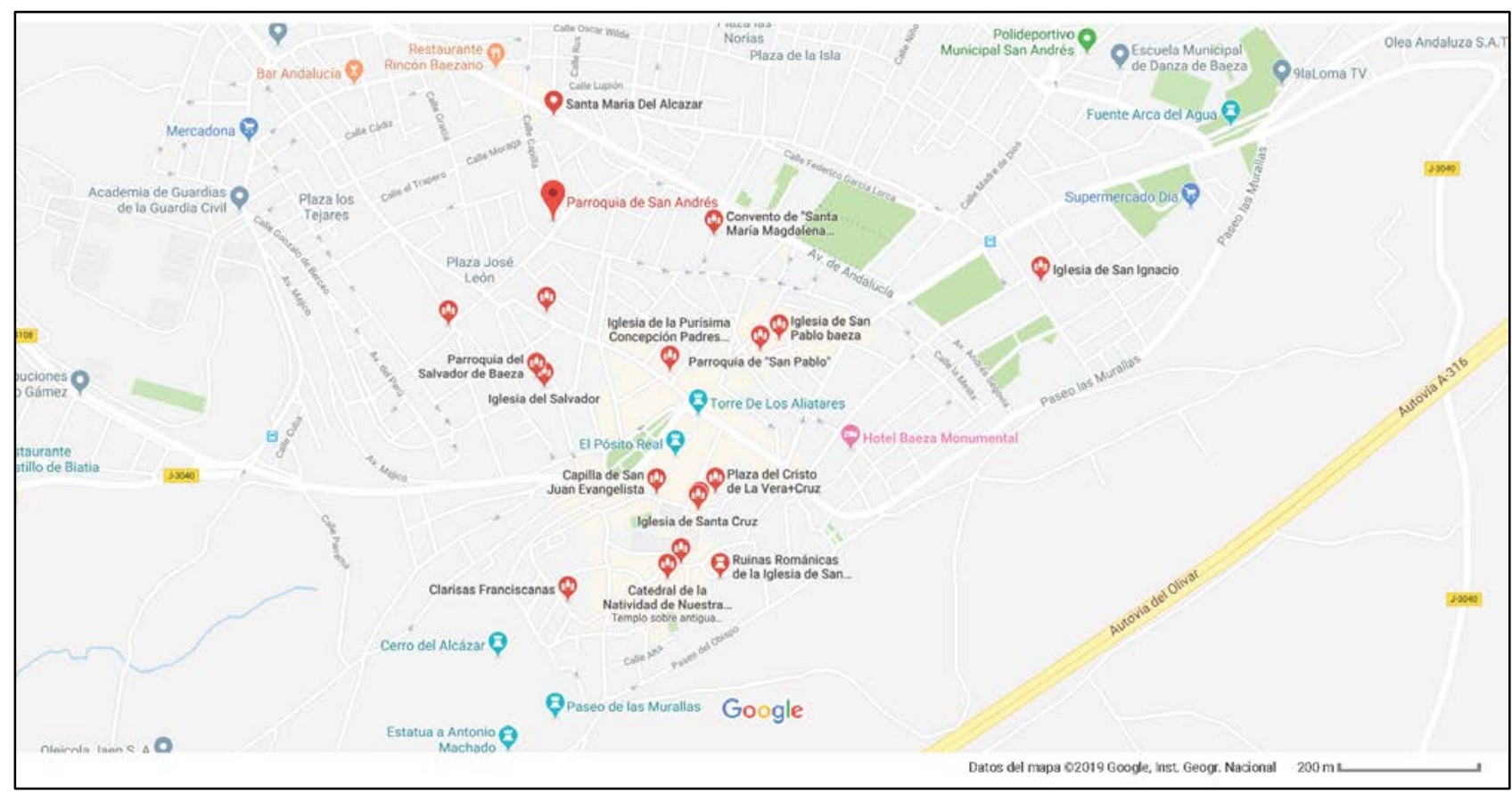

Fuente: Google Maps

Ambas ciudades pertenecen al grupo de Ciudades Patrimonio Mundial desde 2014. Además, realizan un importante esfuerzo de promoción con el objetivo de que los potenciales visitantes conozcan los diferentes recursos y actividades que les ofrecen y decidan realizar el viaje.

Todos estos esfuerzos se ven recompensados mediante el incremento del gasto realizado por los turistas en ambas desde que fueran declaradas Patrimonio de la Humanidad, pasando de un gasto medio diario en 2003 de 74,94 € a uno de 196,80 € en 2012 (Cárdenas-García et al., 2014). Estos datos encuentran su explicación en la consolidación en Úbeda y Baeza como destinos turísticos culturales que han atraído a turistas de alto poder adquisitivo, hecho que ha incrementado el gasto medio realizado en el destino. Pero esto no solo ha afectado al gasto, sino también a la estancia de los visitantes en el destino y al número de turistas, que también han aumentado, aunque en menor medida que el gasto medio diario.

\subsection{Cuestionario}

Para caracterizar al turista que visita las Ciudades del Patrimonio Mundial de Úbeda y Baeza, se han analizado los datos procedentes de 2126 encuestas realizadas en estas ciudades entre junio y septiembre de 2018. La ficha técnica de la encuesta aparece recogida en la Tabla 1.

Para ello, dada la imposibilidad de acotar el objeto de estudio -todos los turistas que visitan estas ciudades durante los meses en los que se realizó la encuesta- y, por tanto, tratándose de una población infinita, se realizó un muestreo aleatorio simple en el que el único criterio de selección fue el de haber pernotado, al menos, una noche en alguna de estas ciudades. Las encuestas se 
realizaron en los lugares más visitados de cada ciudad, como son la Plaza de Santa María en úbeda y la Universidad Internacional de Andalucía Campus Antonio Machado, asentado en el Palacio de Jabalquinto y el Antiguo Seminario Conciliar.

Tabla 1. Ficha técnica de la encuesta

\begin{tabular}{|l|l|}
\hline Población & $\begin{array}{l}\text { Turistas -nacionales y extranjeros- que pernoctan en } \\
\text { una de las ciudades de destino }\end{array}$ \\
\hline Ámbito & Úbeda y Baeza \\
\hline Tipo de encuesta & $\begin{array}{l}\text { Cuestionario estructurado realizado mediante entrevista } \\
\text { personal }\end{array}$ \\
\hline Tamaño de la muestra & 2126 encuestas válidas \\
\hline Error muestral & $2,1 \%$ \\
\hline Nivel de confianza & 95 por $100(p=q=0,50)$ \\
\hline $\begin{array}{l}\text { Periodo del trabajo de } \\
\text { campo }\end{array}$ & Junio, julio, agosto y septiembre de 2018 \\
\hline
\end{tabular}

Fuente: elaboración propia

La encuesta se estructuró en siete bloques, cuatro relativos a los distintos tipos de variables que se van a analizar -socioeconómicas, las relacionadas con las características del viaje, las relacionadas con el destino y las valoraciones y opiniones del turista-, además de incluir tres bloques más relacionados con el presupuesto que el turista dispone para el viaje y el gasto realizado por los turistas en origen y en destino. Se han utilizado distintos tipos de preguntas: abiertas, cerradas dicotómicas y multicotómicas, tanto de respuesta única, como múltiple- y mixtas. Las escalas empleadas para la medición de las variables de estudio han sido también de diferentes tipos: por un lado, una escala de medición de tipo Likert y, por otro, escalas no métricas de carácter nominal, para identificar las categorías u opciones con las que se identifica el comportamiento del entrevistado, en el caso del análisis de variables cualitativas que carecen de significado cuantitativo.

Con las herramientas de la estadística descriptiva, a través del software estadístico SPSS 21.0, se realiza un primer análisis en el que se describen las variables categóricas, utilizando frecuencias y porcentajes. Las variables cuantitativas recogidas se resumen mediante la media, desviación típica, mediana, máximo y mínimo, y se presenta el intervalo de confianza del 95\% para el valor de la media.

\section{Resultados y discusión}

A continuación, se realiza un análisis descriptivo de los cuatro grupos de variables que se incluyeron en el cuestionario -variables sociodemográficas, las relacionadas con las características del viaje, las relacionadas con las características del destino y las variables psicológicas del turista-, así como otros aspectos relacionados con el presupuesto del viaje y los gastos realizados para el mismo. 
En primer lugar, se presentan los resultados de las variables sociodemográficas del cuestionario (Tabla 2).

Tabla 2. Descripción de las variables sociodemográficas del cuestionario

\begin{tabular}{|c|c|c|c|}
\hline & $\mathrm{N}(\%)$ & & $N(\%)$ \\
\hline \multicolumn{2}{|l|}{ Sexo } & \multicolumn{2}{|l|}{ Acompañados por residentes } \\
\hline Hombre & $1077(50,8)$ & No & $2036(95,8)$ \\
\hline Mujer & $1045(49,2)$ & Sí & $90(4,2)$ \\
\hline Perdidos/N & $4 / 2126$ & \multicolumn{2}{|l|}{ Situación laboral } \\
\hline \multicolumn{2}{|l|}{ Edad } & Ocupado & $1392(65,8)$ \\
\hline Menos de 10 años & $162(7,6)$ & Parado & $43(2)$ \\
\hline De 10 a 18 años & $178(8,4)$ & Retirado/Jubilado & $221(10,4)$ \\
\hline De 19 a 29 años & $139(6,6)$ & Labores del hogar & $62(2,9)$ \\
\hline De 30 a 44 años & $381(18)$ & Estudiante & $397(18,8)$ \\
\hline De 45 a 65 años & $1050(49,5)$ & Perdidos/N & $11 / 2126$ \\
\hline Más de 65 años & $212(10)$ & \multicolumn{2}{|l|}{ Donde dormirán esa noche } \\
\hline Perdidos/N & $4 / 2122$ & Úbeda & $1184(56,4)$ \\
\hline \multicolumn{2}{|l|}{ Estudios } & Baeza & $914(43,6)$ \\
\hline $\begin{array}{l}\text { Bachiller Elemental, EGB o ESO } \\
\text { (Graduado Escolar) }\end{array}$ & $237(11,2)$ & Perdidos/N & 0 \\
\hline $\begin{array}{l}\text { Bachiller Superior, BUP, Bachiller } \\
\text { LOGSE, COU, PREU }\end{array}$ & $167(7,9)$ & \multicolumn{2}{|l|}{ Categoría profesional } \\
\hline $\begin{array}{l}\text { FPI, FP grado medio, oficial industrial o } \\
\text { equivalente }\end{array}$ & $133(6,3)$ & Profesional liberal & $492(23,2)$ \\
\hline $\begin{array}{l}\text { FPII, FP grado superior, Maestría } \\
\text { Industrial o equivalente }\end{array}$ & $337(15,9)$ & Empresario & $125(5,9)$ \\
\hline $\begin{array}{l}\text { Diplomatura o tres cursos aprobados } \\
\text { de licenciatura }\end{array}$ & $211(10)$ & Directivo & 0 \\
\hline Licenciatura & $984(46,4)$ & $\begin{array}{l}\text { Funcionario con titulación } \\
\text { universitaria }\end{array}$ & $502(23,7)$ \\
\hline Doctorado, máster & $50(2,4)$ & Mando intermedio & $137(6,5)$ \\
\hline Perdidos/N & $7 / 2126$ & Trabajador cualificado & $304(14,3)$ \\
\hline \multicolumn{2}{|l|}{ Ingresos mensuales } & Trabajador sin cualificar & $50(2,4)$ \\
\hline$<655$ & $491(23,2)$ & Funcionarios sin titulación & $12(0,6)$ \\
\hline $655-900$ & $38(1,8)$ & Otros & $474(22,3)$ \\
\hline $901-1200$ & $239(11,3)$ & Total & $2122(100)$ \\
\hline $1200-1500$ & $344(16,3)$ & Perdidos/N & $4 / 2122$ \\
\hline $1501-1800$ & $377(17,8)$ & \multicolumn{2}{|l|}{ Valores } \\
\hline $1801-2100$ & $335(15,8)$ & Vida cómoda & $1304(61,3)$ \\
\hline $2101-3000$ & $195(9,2)$ & Vida emocionante & $263(12,4)$ \\
\hline$>3000$ & $95(4,5)$ & lgualdad & 0 \\
\hline Perdidos/N & $12 / 2126$ & Libertad & $94(4,4)$ \\
\hline \multicolumn{2}{|l|}{ País de residencia } & Seguridad & $4(0,2)$ \\
\hline España & $1876(88,2)$ & Placer & $6(0,3)$ \\
\hline Extranjero & $250(11,8)$ & Estresante & $415(19,5)$ \\
\hline \multicolumn{2}{|l|}{ Nacionalidad } & Sabiduría & $2(0,1)$ \\
\hline Española & $1884(88,8)$ & Placer & $6(0,3)$ \\
\hline Extranjera & $238(11,2)$ & Estresante & $415(19,5)$ \\
\hline
\end{tabular}

Fuente: elaboración propia

La distribución de encuestados se corresponde prácticamente con un 50 \% de hombres y mujeres. El $50 \%$ de los encuestados tenían entre 45 y 65 años, mayoritariamente con estudios universitarios y con unos ingresos que se sitúan en una franja media-alta -el 49,9\% disponían de ingresos entre 1200 y $2100 €$ al mes, aunque también es cierto que un 23,2 \% manifestaron disponer de menos 
de $655 €-$. Respecto al país de residencia, los encuestados son mayoritariamente de España $(88,2 \%)$, característica que comparte similar porcentaje con la nacionalidad de los mismos.

En lo relacionado con la situación laboral, el grueso lo componen los ocupados (65,8 \%), seguido de los estudiantes (18,8\%). Dentro de la categoría profesional de dichos ocupados, destacan el profesional liberal $(23,2 \%)$ y el funcionario con titulación universitaria $(23,7 \%)$. Por otro lado, se observa que solamente un 4,2 \% de los encuestados realizan el viaje acompañados de personas que residen en úbeda o Baeza. Y, por otra parte, un 56,4\% de los encuestados pasan la noche en Úbeda, mientras que un 43,6\% lo hace en Baeza. En último lugar, se puede apreciar que el valor predominante en los encuestados es el de una vida cómoda (61,3\%), proseguido de una vida estresante $(19,5 \%)$ y una vida emocionante $(12,4 \%)$.

Seguidamente (Tabla 3), se recoge la descripción de las respuestas a las variables relacionadas con las características del viaje, incluyendo frecuencia y porcentaje por categoría de respuesta, así como media, desviación típica, intervalo de confianza al $95 \%$, mediana y máximo y mínimo, en el caso de las variables continuas.

Tabla 3. Descripción de las variables correspondiente a las características del viaje

\begin{tabular}{|c|c|c|c|c|c|}
\hline & $\mathrm{N}(\%)$ & & $\mathrm{N}(\%)$ & & $\mathrm{N}(\%)$ \\
\hline \multicolumn{2}{|l|}{ Recomendación } & \multicolumn{2}{|l|}{ Internet transporte } & \multicolumn{2}{|c|}{$\begin{array}{l}\text { Cómo conoció este destino: agencia } \\
\text { de viajes }\end{array}$} \\
\hline No & $2(0,1)$ & Consulta online & $63(3)$ & No & $2043(96,1)$ \\
\hline $\mathrm{Si}$ & $2124(99,9)$ & Reserva online & $8(0,4)$ & Sí & $83(3,9)$ \\
\hline \multicolumn{2}{|l|}{ Modalidad de viaje } & Comprar online & $95(4,5)$ & \multicolumn{2}{|l|}{ Amigos o familiares } \\
\hline Solo & $19(0,9)$ & No utilizó & $1960(92,2)$ & No & $1392(65,5)$ \\
\hline Pareja sin niños & $673(31,7)$ & \multicolumn{2}{|l|}{ Internet alojamiento } & Sí & $734(34,5)$ \\
\hline Pareja con niños & $666(31,3)$ & Consulta online & $18(0,8)$ & \multicolumn{2}{|l|}{ Experiencia propia } \\
\hline $\begin{array}{l}\text { Familia que reside con } \\
\text { ancestros }\end{array}$ & $63(3)$ & Reserva online & $8(0,4)$ & No & $1710(80,4)$ \\
\hline Amigos & $623(29,3)$ & Comprar online & $1931(90,8)$ & Sí & $416(19,6)$ \\
\hline Otros & $82(3,9)$ & No utilizó & $169(7,9)$ & \multicolumn{2}{|l|}{ Iniciativa propia } \\
\hline \multicolumn{2}{|l|}{ Regresaría } & \multicolumn{2}{|l|}{ Internet restaurantes } & No & $1931(90,8)$ \\
\hline Sí & $1806(87,5)$ & Consulta online & $1014(48,1)$ & Sí & $195(9,2)$ \\
\hline No & $257(12,5)$ & Reserva online & $25(1,2)$ & \multicolumn{2}{|l|}{ Guías turísticas } \\
\hline Perdidos & $63 / 2063$ & Comprar online & $24(1,1)$ & No & $2074(97,6)$ \\
\hline \multicolumn{2}{|l|}{$\mathrm{N}^{\circ}$ visitas } & No utilizó & $1043(49,5)$ & Sí & $52(2,4)$ \\
\hline Ninguna & $1285(60,4)$ & Perdidos/Total & $20 / 2126$ & \multicolumn{2}{|l|}{ Ofertas y catálogos } \\
\hline Una vez & $\begin{array}{r}161 \\
(7,6)\end{array}$ & \multicolumn{2}{|l|}{ Internet lugares que visitar } & No & $2126(100)$ \\
\hline Dos veces & $280(13,2)$ & Consulta online & $1167(54,9)$ & \multicolumn{2}{|l|}{ Internet } \\
\hline Tres veces & $\begin{array}{r}119 \\
(5,6) \\
\end{array}$ & Reserva online & $15(0,7)$ & No & $2024(95,2)$ \\
\hline Cuatro a Siete veces & $\begin{array}{r}83 \\
(3,9) \\
\end{array}$ & No utilizó & $942(44,4)$ & Sí & $102(4,8)$ \\
\hline Más de siete veces & $\begin{array}{r}198 \\
(9,3)\end{array}$ & Perdidos & $2 / 2126$ & & \\
\hline
\end{tabular}


Tabla 3. Continuación

\begin{tabular}{|c|c|c|c|c|c|}
\hline \multirow{3}{*}{ Forma de organizar el viaje } & \multirow[t]{3}{*}{$\mathrm{N}(\%)$} & \multirow{3}{*}{\multicolumn{2}{|c|}{ Internet entradas }} & \multirow{2}{*}{\begin{tabular}{l|l} 
& $\mathrm{N}(\%)$ \\
Publicidad en prensa
\end{tabular}} & \multirow[t]{2}{*}{$\mathrm{N}(\%)$} \\
\hline & & & & & \\
\hline & & & & No & $2122(99,8)$ \\
\hline Particular & $2060(97,3)$ & Consulta online & $601(28,3)$ & Sí & $4(0,2)$ \\
\hline Agencia de Viajes & $58(2,7)$ & No utilizó & $1524(71,7)$ & \multicolumn{2}{|c|}{$\begin{array}{l}\text { Saber que era Ciudad Patrimonio } \\
\text { Humanidad }\end{array}$} \\
\hline Perdidos/Total & $8 / 2126$ & Perdidos/Total & $1 / 2126$ & No & $1620(76,2)$ \\
\hline \multicolumn{2}{|l|}{ Alojamiento } & \multicolumn{2}{|c|}{ Internet alquiler vehículo } & Sí & $506(23,8)$ \\
\hline Hotel 3* & $1068(50,8)$ & Consulta online & $63(3)$ & \multicolumn{2}{|c|}{ Otros } \\
\hline Hotel $1^{\star} \circ 2^{\star}$ & $602(28,7)$ & Reserva online & $14(0,7)$ & No & $2054(96,6)$ \\
\hline Hostal o pensión & $132(6,3)$ & Comprar online & $109(5,1)$ & Sí & $72(3,4)$ \\
\hline Camping & $6(0,3)$ & No utilizó & $1940(91,3)$ & \multicolumn{2}{|c|}{ Tipo de transporte: coche propio } \\
\hline Albergue/Residencia & $2(0,1)$ & \multicolumn{2}{|l|}{ Internet otros } & No & $228(10,7)$ \\
\hline Aparta hotel $4^{*} \circ 5^{*}$ & $6(0,3)$ & Consulta online & $530(24,9)$ & Sí & $1898(89,3)$ \\
\hline Aparta hotel $3^{*}$ & $20(1)$ & Comprar online & $4(0,2)$ & \multicolumn{2}{|c|}{ Coche alquilado } \\
\hline Aparta hotel $1^{\star} \circ 2^{*}$ & $7(0,3)$ & No utilizó & $1592(74,9)$ & No & $1972(92,8)$ \\
\hline $\begin{array}{l}\text { Casa o Apartamentos } \\
\text { Rurales }\end{array}$ & $49(2,3)$ & \multicolumn{2}{|c|}{+2} & Sí & $154(7,2)$ \\
\hline $\begin{array}{l}\text { Apartamentos o casas } \\
\text { alquiladas }\end{array}$ & $73(3,5)$ & \multicolumn{2}{|c|}{ Impedimento de pago } & \multicolumn{2}{|c|}{ Autobús discrecional } \\
\hline $\begin{array}{l}\text { Casa/apartamentos de } \\
\text { amigos o familiares }\end{array}$ & $136(6,5)$ & No & $2112(99,3)$ & No & $2068(97,3)$ \\
\hline Perdidos/Total & $25 / 2126$ & Sí & $14(0,7)$ & Sí & $58(2,7)$ \\
\hline \multicolumn{2}{|l|}{ Visita más lugares } & \multicolumn{2}{|c|}{ Uso de redes sociales } & \multicolumn{2}{|c|}{ Autobús de línea } \\
\hline No & $1318(62)$ & No & $1724(81,1)$ & No & $2112(99,3)$ \\
\hline Sí & $808(38)$ & Sí & $402(18,9)$ & Si & $14(0,7)$ \\
\hline \multicolumn{2}{|l|}{ Tipo de reserva realizada } & \multicolumn{2}{|c|}{ Uso de portales institucionales } & \multicolumn{2}{|c|}{ Motocicleta } \\
\hline Ninguna & $160(7,5)$ & No & $1909(89,8)$ & No & $2126(100)$ \\
\hline $\begin{array}{l}\text { Organizado todo por } \\
\text { agencia }\end{array}$ & $5(0,2)$ & Sí & $217(10,2)$ & \multicolumn{2}{|l|}{ Tren } \\
\hline $\begin{array}{l}\text { Organizado alojamiento por } \\
\text { agencia }\end{array}$ & $77(3,6)$ & \multicolumn{2}{|c|}{ Uso de buscadores } & No & $2108(99,2)$ \\
\hline Alojamiento particular & $1757(82,6)$ & No & $1562(73,5)$ & Sí & $18(0,8)$ \\
\hline $\begin{array}{l}\text { Trasporte más alojamiento } \\
\text { por agencia }\end{array}$ & $27(1,3)$ & Sí & $564(26,5)$ & \multicolumn{2}{|c|}{ Bicicleta } \\
\hline $\begin{array}{l}\text { Trasporte más alojamiento } \\
\text { por particular }\end{array}$ & $100(4,7)$ & \multicolumn{2}{|l|}{ Google Maps } & No & $2126(100)$ \\
\hline \multicolumn{2}{|l|}{ Internet transporte y alojamiento } & No & $1841(86,6)$ & Barce & \\
\hline Consulta Online & $42(2)$ & Sí & $285(13,4)$ & No & $2126(100)$ \\
\hline Reserva Online & $13(0,6)$ & \multicolumn{2}{|l|}{ Apps } & \multicolumn{2}{|c|}{ Avión } \\
\hline Comprar Online & $44(2,1)$ & No & $2126(100)$ & No & $1967(92,5)$ \\
\hline No utilizó & $2027(95,3)$ & Otros & & Sí & $159(7,5)$ \\
\hline & & No & $1433(67,4)$ & & \\
\hline & & Sí & $693(32,6)$ & & \\
\hline
\end{tabular}

\begin{tabular}{|l|c|c|c|c|c|c|c|c|}
\cline { 5 - 8 } \multicolumn{1}{c|}{} & \multirow{2}{*}{$\mathrm{N}$} & \multirow{2}{*}{ Media } & \multirow{2}{*}{$\begin{array}{c}\text { Desviación } \\
\text { típica }\end{array}$} & \multicolumn{2}{c|}{ Intervalo de confianza } & \multirow{2}{*}{ Mediana } & \multirow{2}{*}{ Mín } & \multirow{2}{*}{ Max } \\
\hline $\mathrm{N}^{\circ}$ de días del viaje & 2126 & 2,83 & 2,546 & 2,73 & 2,94 & 2 & 1 & 20 \\
\hline $\begin{array}{l}N^{\circ} \text { de personas del } \\
\text { viaje }\end{array}$ & 2126 & 4,63 & 5,287 & 4,43 & 4,87 & 4 & 1 & 50 \\
\hline$N^{\circ}$ de noches del viaje & 2126 & 3,52 & 3,807 & 3,37 & 3,69 & 2 & 1 & 20 \\
\hline
\end{tabular}

Fuente: elaboración propia

Prácticamente la totalidad de los turistas recomendaría la ciudad en la que se le ha encuestado como destino de viaje. Las modalidades más comunes de viaje han sido las de parejas con y sin niños, en torno a un 31 \% cada una, seguidas por amigos (29,3\%). Se puede observar que los encuestados son, en su mayoría, nuevos en las ciudades de destino (el 60,4\% jamás las había 
visitado), que predominantemente no visitan más lugares (62\%) y que, frente a la posibilidad de regresar a Úbeda o Baeza, la afirmación presenta el 87,5\% de las opiniones.

Respecto a la organización del viaje, casi íntegramente ha sido de forma particular, hospedándose la mitad de los encuestados en hoteles de 3 estrellas (seguido por los hoteles de 2 o 1 estrellas) y utilizando Internet de forma escasa para el transporte, alquiler de vehículos y entradas a lugares culturales, a diferencia del uso para el alojamiento, para el que un 90,8\% de los encuestados realizó una compra online. El uso de Internet para los restaurantes muestra casi un $50 \%$ de encuestados que realizan una consulta. De manera similar ocurre con los lugares que visitar, con $54,9 \%$ de los encuestados que manifiesta haber utilizado Internet para consultar respecto a ellos. También cabe destacar que, casi en la totalidad de los encuestados, no han existido problemas de pago, es decir, en el momento en el que fueron a realizar el pago de los servicios y productos adquiridos en el destino pudieron realizar el pago en metálico, en tarjeta e inclusive a través del uso de cheques de regalo.

En lo relativo a la forma en la que los encuestados conocieron el destino, se contempla un mayor porcentaje de éstos a través de amigos o familiares, por la experiencia propia y por saber que es Patrimonio de la Humanidad (34,5\%, 19,6 \% y 23,8 \% respectivamente); mientras que, en el otro extremo, se encuentran aquellos menos relevantes, como la publicidad en prensa $(0,2 \%)$ y ofertas y catálogos (0\%). Asimismo, en los recursos que utilizaron para conocer la ciudad, existe una tendencia de uso reducida por parte de los encuestados, con valores de $26,5 \%$ en buscadores o $18,9 \%$ en redes sociales. Por otra parte, respecto al tipo de transporte utilizado, destaca el predominio del coche propio (89,3\%).

Por la parte de las variables cuantitativas, se puede observar que la duración media de los viajes gira en torno a 2-3 días, siendo realizado por 4-5 personas y pasando entre 2 y 3 noches en los destinos. La existencia de una variabilidad tan acusada radica en el hecho de la heterogeneidad de los encuestados, puesto que, a pesar de situar los valores medios en dichos intervalos, existen mínimos y máximos de 1 y 20 (en el caso de días/noches) y de 1 y 50 (en el caso de personas).

En tercer lugar, se exponen los resultados descriptivos de las variables relacionadas con las características del destino (Tabla 4).

Tabla 4. Descripción de las variables relacionadas con las características del destino

\begin{tabular}{|l|r|}
\cline { 2 - 2 } \multicolumn{1}{c|}{} & $\mathrm{N}(\%)$ \\
\hline Acompañados por personas residentes & $2036(95,8)$ \\
\hline No & $90(4,2)$ \\
\hline Sí & $2(0,1)$ \\
\hline Encontrar lo que esperaba & $2102(98,9)$ \\
\hline No & $22(1)$ \\
\hline Sí &
\end{tabular}


Tabla 4. Continuación

\begin{tabular}{|l|r|}
\hline Actividades que ha realizado: visita puntual & $2(0,1)$ \\
\hline No & $2124(99,9)$ \\
\hline Sí & $2067(97,2)$ \\
\hline Visita museos & $59(2,8)$ \\
\hline No & $236(11,1)$ \\
\hline Sí & $1890(88,9)$ \\
\hline Rutas guiadas & $1920(90,3)$ \\
\hline No & $206(9,7)$ \\
\hline Sí & \\
\hline Ruta cultural & $81(3,8)$ \\
\hline No & $2045(96,2)$ \\
\hline Sí & \\
\hline Ir de tapas & $2045(96,2)$ \\
\hline No & $81(3,8)$ \\
\hline Sí & \\
\hline Compras de artesanía & $2102(98,9)$ \\
\hline No & $24(1,1)$ \\
\hline Sí & \\
\hline Comprar libros & $1134(53,3)$ \\
\hline No & $992(46,7)$ \\
\hline Sí & $1784(83,9)$ \\
\hline Ir de copas & $342(16,1)$ \\
\hline No
\end{tabular}

Fuente: elaboración propia

La totalidad de los encuestados han manifestado encontrar durante su visita lo que esperaban, por lo que se concluye que la visita ha satisfecho sus expectativas. Sobre las actividades en los lugares de destino, la visita puntual $(99,9 \%)$, las rutas guiadas $(88,9 \%)$, ir de tapas $(96,2 \%)$ e ir de copas, en menor grado (46,7\%), son las principales actividades llevadas a cabo por los encuestados, a diferencia de comprar libros (1,1\%), visitar museos (2,8\%), comprar artesanía $(3,8 \%)$ o rutas culturales $(9,7 \%)$.

En la Tabla 5, se describen las respuestas a las cuestiones relacionadas con las variables psicológicas de los turistas y a los motivos que propiciaron el viaje, incluyendo frecuencia y porcentaje por categoría de respuesta, así como media, desviación típica, intervalo de confianza al $95 \%$, mediana y máximo y mínimo, en el caso de las variables continuas. 
Tabla 5. Descripción de las variables psicológicas del turista y motivos que propiciaron el viaje

\begin{tabular}{|c|c|c|c|}
\hline & $\mathrm{N}(\%)$ & & $\mathrm{N}(\%)$ \\
\hline \multicolumn{2}{|c|}{ Mente abierta } & \multicolumn{2}{|c|}{ Motivo de viaje: Ocio/Nacaciones } \\
\hline No & $997(46,9)$ & No & $77(3,6)$ \\
\hline Sí & $1129(53,1)$ & Sí & $2049(96,4)$ \\
\hline \multicolumn{2}{|c|}{ Alegre } & \multicolumn{2}{|c|}{ Negocios/Trabajo } \\
\hline No & $1494(70,3)$ & No & $2117(99,6)$ \\
\hline Sí & $632(29,7)$ & Sí & $9(0,4)$ \\
\hline \multicolumn{2}{|c|}{ Limpio } & \multicolumn{2}{|l|}{ Reuniones } \\
\hline No & $2086(98,1)$ & No & $2110(99,2)$ \\
\hline Sí & $40(1,9)$ & Sí & $16(0,8)$ \\
\hline \multicolumn{2}{|c|}{ Valiente } & \multicolumn{2}{|l|}{ Salud } \\
\hline No & $1943(91,4)$ & No & $2126(100)$ \\
\hline Sí & $183(8,6)$ & \multicolumn{2}{|c|}{ Familiares/Amigos } \\
\hline \multicolumn{2}{|c|}{ Honesto } & No & $2072(97,5)$ \\
\hline No & $2036(95,8)$ & Sí & $54(2,5)$ \\
\hline Sí & $90(4,2)$ & \multicolumn{2}{|c|}{ Eventos deportivos } \\
\hline \multicolumn{2}{|c|}{ Cariñoso } & No & $2126(100)$ \\
\hline No & $2067(97,2)$ & \multicolumn{2}{|l|}{ Eventos culturales } \\
\hline Sí & $59(2,8)$ & No & $2126(100)$ \\
\hline \multicolumn{2}{|c|}{ Obediente } & \multicolumn{2}{|l|}{ Gastronomía } \\
\hline No & $2122(99,8)$ & No & $2126(100)$ \\
\hline Sí & $4(0,2)$ & \multicolumn{2}{|l|}{ Estudios } \\
\hline \multicolumn{2}{|c|}{ Cortés } & No & $2118(99,6)$ \\
\hline No & $2104(99)$ & Sí & $8(0,4)$ \\
\hline Sí & $22(1)$ & \multicolumn{2}{|c|}{ Grado de satisfacción del viaje } \\
\hline \multicolumn{2}{|c|}{ Responsable con los demás } & Muy positivo & $1426(67,3)$ \\
\hline No & $2112(99,3)$ & Positivo & $653(30,8)$ \\
\hline Sí & $14(0,7)$ & Esperado & $33(1,6)$ \\
\hline \multicolumn{2}{|c|}{ Sereno } & Negativo & $4(0,2)$ \\
\hline No & $2119(99,7)$ & Muy negativo & $2(0,1)$ \\
\hline Sí & $7(0,3)$ & Perdidos/Total & $8 / 2126$ \\
\hline
\end{tabular}

\begin{tabular}{|c|c|c|c|c|c|c|c|c|c|}
\hline & \multirow[t]{2}{*}{$\mathrm{N}$} & \multirow[t]{2}{*}{ Media } & \multirow{2}{*}{$\begin{array}{l}\text { Desviación } \\
\text { típica }\end{array}$} & \multicolumn{2}{|c|}{$\begin{array}{l}\text { Intervalo de } \\
\text { Confianza }\end{array}$} & \multirow[t]{2}{*}{ Mediana } & \multirow[t]{2}{*}{ Mín } & \multirow[t]{2}{*}{ Max } & \multirow{2}{*}{$\begin{array}{c}\text { Perdidos } \\
\text { / Total }\end{array}$} \\
\hline & & & & Inferior & Superior & & & & \\
\hline Actividades culturales & 2126 & 8,27 & 0,93 & 8,23 & 8,31 & 8,00 & 4,00 & 10,00 & \\
\hline Alojamiento & 2112 & 8,31 & 0,97 & 8,27 & 8,35 & 8,00 & 4,00 & 10,00 & $14 / 2126$ \\
\hline Asistencia Sanitaria & 1987 & 7,99 & 0,96 & 7,94 & 8,03 & 8,00 & 4,00 & 10,00 & $139 / 2126$ \\
\hline Atención y trato & 2126 & 7,92 & 1,30 & 7,86 & 7,97 & 8,00 & 2,00 & 10,00 & \\
\hline Relación calidad/precio & 2126 & 8,09 & 1,11 & 8,04 & 8,14 & 8,00 & 3,00 & 10,00 & \\
\hline $\begin{array}{l}\text { Carreteras y } \\
\text { comunicaciones }\end{array}$ & 2126 & 6,80 & 1,71 & 6,73 & 6,87 & 7,00 & 1,00 & 10,00 & \\
\hline Entornos urbanos & 2126 & 7,91 & 1,17 & 7,86 & 7,96 & 8,00 & 1,00 & 10,00 & \\
\hline Información turística & 2126 & 6,67 & 1,87 & 6,58 & 6,75 & 7,00 & 3,00 & 10,00 & \\
\hline $\begin{array}{l}\text { Posibilidades de acceso } \\
\text { a internet }\end{array}$ & 2124 & 7,78 & 1,08 & 7,73 & 7,82 & 8,00 & 4,00 & 10,00 & $2 / 2126$ \\
\hline Limpieza & 2114 & 7,86 & 1,22 & 7,80 & 7,91 & 8,00 & 1,00 & 10,00 & $12 / 2126$ \\
\hline Ocio/Diversión & 2126 & 8,13 & 1,06 & 8,09 & 8,18 & 8,00 & 4,00 & 10,00 & \\
\hline Paisajes & 2126 & 8,57 & 0,98 & 8,53 & 8,61 & 9,00 & 4,00 & 10,00 & \\
\hline Restauración & 2126 & 8,04 & 1,15 & 8,00 & 8,09 & 8,00 & 4,00 & 10,00 & \\
\hline Seguridad ciudadana & 2016 & 7,86 & 1,05 & 7,81 & 7,90 & 8,00 & 4,00 & 10,00 & $110 / 2126$ \\
\hline Señalización turística & 2126 & 6,00 & 1,90 & 5,93 & 6,08 & 5,00 & 2,00 & 10,00 & \\
\hline Tráfico/Aparcamiento & 2126 & 6,33 & 1,96 & 6,25 & 6,42 & 6,00 & 3,00 & 10,00 & \\
\hline Tranquilidad (ruidos) & 2126 & 8,35 & 0,99 & 8,31 & 8,39 & 9,00 & 4,00 & 10,00 & \\
\hline Transporte público & 2012 & 8,04 & 1,02 & 7,99 & 8,08 & 8,00 & 3,00 & 10,00 & $114 / 2126$ \\
\hline
\end{tabular}

Fuente: elaboración propia

Como datos más significativos, un $53,1 \%$ de los encuestados consideran que tienen una mentalidad abierta, junto con el 29,7\% que poseen personalidad alegre. Menor representación tienen las demás cualidades, como sereno, responsable o cortés. En relación al motivo de viaje, 
predomina el ocio y vacaciones $(96,4 \%)$, seguido, con gran diferencia, por visitas a familiares y amigos $(2,5 \%)$, siendo el resto de categorías poco relevantes. A propósito del grado de satisfacción del viaje, un mayor porcentaje de encuestados afirman tener un grado muy positivo o positivo (67,3\% y $30,8 \%$, respectivamente).

En lo relacionado con las variables cuantitativas, hay que considerar la calificación que otorgan los encuestados a una serie de aspectos relacionados con los destinos analizados. Dichos aspectos obtienen una mayor satisfacción en los valores cercanos a 10. En consecuencia, es percibido que el alojamiento, las actividades culturales, la relación calidad/precio, el ocio y diversión, los paisajes, la restauración, la tranquilidad y el transporte público presentan una media mayor a 8, siendo, por tanto, los rasgos mejor valorados por los encuestados. Por la parte contraria, hay que tener en cuenta la señalización/información turística y el tráfico/carreteras, con mayor insatisfacción por parte de los visitantes.

Respecto a los aspectos económicos del viaje, como datos más relevantes, cabe destacar que el presupuesto medio por persona y día del que disponen los turistas es de 129,43 € (Tabla 6). Aunque el valor que se más se repite en la encuesta (la mediana) es de un presupuesto de $110 €$. Por otra parte, la mayoría de los turistas que acudieron a Úbeda y Baeza $(99,2 \%)$ no realizaron pagos en su lugar de residencia relacionados con el viaje y, además, en un $100 \%$ no adquirieron paquetes turísticos (Tabla 7).

Tabla 6. Media del presupuesto total del viaje

\begin{tabular}{|c|c|c|c|c|c|c|c|c|c|}
\hline & \multirow[t]{2}{*}{$N$} & \multirow{2}{*}{ Media } & \multirow{2}{*}{$\begin{array}{l}\text { Desviación } \\
\text { típica }\end{array}$} & \multicolumn{2}{|c|}{$\begin{array}{c}95 \% \text { Intervalo de } \\
\text { Confianza }\end{array}$} & \multirow[t]{2}{*}{ Mediana } & \multirow[t]{2}{*}{ Mín. } & \multirow[t]{2}{*}{ Max. } & \multirow{2}{*}{$\begin{array}{l}\text { Perdidos } \\
\text { / Total }\end{array}$} \\
\hline & & & & Inferior & Superior & & & & \\
\hline $\begin{array}{l}\text { Presupuesto } \\
\text { total del viaje }\end{array}$ & 2118 & 129,43 & 60,15 & 126,67 & 132,26 & 110,00 & 30,00 & 430,00 & $8 / 2126$ \\
\hline
\end{tabular}

Fuente: elaboración propia

Tabla 7. Porcentajes del tipo de gastos realizados en el origen

\begin{tabular}{|l|r|}
\cline { 2 - 2 } \multicolumn{1}{c|}{} & N (\%) \\
\hline Pagos en su lugar de residencia \\
\hline No & $2110(99,2)$ \\
\hline Sí & $16(0,8)$ \\
\hline Paquete turístico & $2126(100)$ \\
\hline No &
\end{tabular}

Fuente: elaboración propia

Finalmente, en lo que respecta al tipo de gasto realizado por los turistas (Tabla 8), cabe destacar que la principal partida de gasto es la de alojamiento, en la que los encuestados realizan un gasto 
medio diario de 50,47 €, seguida de la compras en alimentación $(48,81 €)$, alquiler de vehículos $(35,95 €)$, comida $(30,94 €)$ y transporte en el destino $(29,45 €)$. En cualquier caso, hay que significar que el 85,75\% de los encuestados no realiza compras en alimentación, por lo que ésta es una partida de gasto más bien testimonial, frente a la partida de comida, que sí utilizan el $98,35 \%$ de los encuestados. A visitas organizadas y excursiones solo se dedican 14,58 $\in$. Y llama poderosamente la atención que el 93,93 \% de los encuestados no realizan ningún tipo de gasto en regalos, souvenirs, etc., mientras que el 61,71\% no realiza gastos en ocio (museos, exposiciones, actividades deportivas, etc.).

Tabla 8. Descripción de las variables de gasto consideradas en el estudio

\begin{tabular}{|c|c|c|c|c|c|c|c|c|c|}
\hline & \multirow{2}{*}{$\begin{array}{c}N \text { gasto } \\
>0\end{array}$} & \multirow{2}{*}{ Media } & \multirow{2}{*}{$\begin{array}{l}\text { Desviación } \\
\text { Típica }\end{array}$} & \multicolumn{2}{|c|}{$\begin{array}{c}\text { 95\% Intervalo de } \\
\text { confianza }\end{array}$} & \multirow{2}{*}{ Mediana } & \multirow{2}{*}{ Mín } & \multirow{2}{*}{ Max } & \multirow{2}{*}{$\begin{array}{c}\text { No gasto } 0 \\
/ \text { Total }\end{array}$} \\
\hline & & & & $\begin{array}{l}\text { Límite } \\
\text { Inferior }\end{array}$ & $\begin{array}{l}\text { Limite } \\
\text { Superior }\end{array}$ & & & & \\
\hline Alojamiento & 1982 & 50,47 & 17,49 & 49,67 & 51,20 & 50,00 & 10,00 & 125,00 & $144 / 2126$ \\
\hline $\begin{array}{l}\text { Compras en } \\
\text { alimentación }\end{array}$ & 303 & 48,81 & 44,62 & 44,07 & 54,09 & 30,00 & 5,00 & 200,00 & $1823 / 2126$ \\
\hline $\begin{array}{l}\text { Visitas organizadas, } \\
\text { excursiones }\end{array}$ & 1918 & 14,58 & 2,47 & 14,47 & 14,69 & 15,00 & 5,00 & 30,00 & $208 / 2126$ \\
\hline $\begin{array}{l}\text { Ocio (museos, } \\
\text { exposiciones, } \\
\text { actividades } \\
\text { deportivas, ...) }\end{array}$ & 814 & 21,17 & 15,23 & 20,17 & 22,27 & 20,00 & 5,00 & 70,00 & $1312 / 2126$ \\
\hline $\begin{array}{l}\text { Otras compras } \\
\text { (regalos, souvenirs, } \\
\text { etc.) }\end{array}$ & 129 & 22,74 & 13,83 & 20,49 & 25,08 & 20,00 & 5,00 & 75,00 & $1997 / 2126$ \\
\hline $\begin{array}{l}\text { Transporte en el } \\
\text { destino }\end{array}$ & 1273 & 29,45 & 30,73 & 27,80 & 31,12 & 20,00 & 5,00 & 250,00 & $853 / 2126$ \\
\hline $\begin{array}{l}\text { Alquiler de } \\
\text { vehículos }\end{array}$ & 132 & 35,95 & 14,46 & 33,41 & 38,64 & 42,50 & 10,00 & 60,00 & $1994 / 2126$ \\
\hline Comidas & 2091 & 30,94 & 10,75 & 30,49 & 31,40 & 30,00 & 5,00 & 100,00 & $35 / 2126$ \\
\hline Otros & 418 & 11,77 & 11,64 & 10,60 & 13,00 & 10,00 & 1,00 & 90,00 & $1708 / 2126$ \\
\hline
\end{tabular}

Fuente: elaboración propia

Puede decirse, por tanto, que la caracterización del turista que visita Úbeda y Baeza es muy similar a la identificada por la literatura científica y descrita en el apartado anterior.

A grandes rasgos, el perfil del turista analizado en este trabajo coincide con lo establecido en la literatura científica en lo que se refiere a la franja de edad, la ocupación y niveles educativos, la nacionalidad, la pernoctación media en hoteles, la razón por la que visitan el destino, el nivel general de satisfacción, que viaja en pareja por ocio y vacaciones y que las actividades que realiza en destino son, principalmente, visitas y rutas turísticas. Aunque las compras son una actividad destacada del turista en CPH (Morales-Fernández \& Lanquar, 2014), no lo son en el caso del turista que visita Úbeda y Baeza.

Sin embargo, destaca que los ingresos percibidos por los turistas que eligen Úbeda y Baeza como destino son inferiores a la renta del turista de $\mathrm{CPH}$ caracterizado por la literatura científica, que lo enmarca en niveles de renta medio-altos. 
Respecto a la satisfacción con determinados aspectos del destino, sí existen diferencias entre los destinos analizados y otros expuestos por la literatura científica. Balcar y Pearce (1996) centraron su estudio en la costa oeste de Nueva Zelanda, donde los turistas mostraron mayor nivel de satisfacción con la facilidad de acceso a los destinos, mientras que el tráfico y las carreteras son los ítems en los que menos satisfacción muestran los turistas de Úbeda y Baeza. En lo que sí coinciden es con la valoración a la relación calidad-precio. Gregori y Jarauta (2015) analizaron el turista que visita San Cristóbal de la Laguna y Córdoba (España), en los que los turistas están muy satisfechos con la información turística, todo lo contrario que los turistas de Úbeda y Baeza, aunque sí coinciden con la valoración dada a la gastronomía y restauración.

A tenor de los resultados obtenidos, el turista que visita Úbeda y Baeza no suele planificar su viaje, lo cual discrepa con el perfil del turista establecido por la mayor parte de la literatura científica (Correia et al., 2010, Gregori \& Jarauta, 2015; Junta de Andalucía, 2010; Marrero-Rodríguez \& Abdul-Jalbar, 2012), a excepción de Balcar \& Pearce (1996). Por otra parte, nuestros resultados coinciden con el trabajo realizado por la Junta de Andalucía (2010), al reconocer el empleo de Internet como clave para realizar reservas.

En materia de gasto, nuestro estudio refleja un turista que gasta más que el analizado por la literatura científica (Junta de Andalucía, 2010; Troitiño \& Troitiño, 2009), aunque sí coincide la distribución del gasto en alojamiento y comida, siendo en Úbeda y Baeza el gasto realizado en alquiler de vehículos más alto que el de comida. Se puede alcanzar la misma conclusión en materia de gasto, al comparar este estudio con el realizado por Prada-Trigo et al., (2015), pero, en este caso, el estudio se realizó en Cuenca (Ecuador), donde el nivel de precios y el poder adquisitivo es inferior.

\section{Conclusiones}

Que un destino sea CPH implica que cuenta con una serie de recursos culturales y patrimoniales que lo hacen merecedor de dicho reconocimiento. La gestión adecuada de estos recursos permite al destino beneficiarse de los efectos positivos de un turismo de calidad, generando puestos de trabajo, promoviendo la actividad local, generando ingresos, etc. (UNESCO, 2014) y de unos recursos extraordinarios, fruto de un mayor gasto turístico, para la conservación del patrimonio, fruto de ese mayor gasto turístico.

El comportamiento, motivaciones e intereses de los turistas a la hora de visitar un destino están fundamentados en las características -sociodemográficas y psicológicas- de estos. En el caso de las $\mathrm{CPH}$, existe un vínculo particular entre el turista cultural y el destino, materializado en la percepción de éste en función del patrimonio que los gestores de estos destinos deben ser capaces de entender y tener en cuenta a la hora de desarrollar políticas turísticas. 
Por tanto, es fundamental para la gestión de un destino conocer estas motivaciones, intereses y percepciones de los turistas, es decir, el perfil del turista en sus diferentes dimensiones, de manera que sea posible satisfacer las necesidades de estos bajo los mayores niveles de satisfacción posibles.

La literatura científica ha dedicado un gran esfuerzo para determinar el perfil del turista que visita diferentes $\mathrm{CPH}$, coincidiendo la mayoría en una serie de variables. En un principio, el análisis se centraba en el estudio de las características sociodemográficas, pero con el tiempo ha ido surgiendo la necesidad de ir más allá y conocer cómo se comporta el turista antes y durante la visita, así como entender cuáles son sus intereses, basados en sus motivaciones y satisfacción con el viaje.

Nuestro estudio perfila el turista que visita úbeda y Baeza como españoles, satisfechos con el destino, de entre 45 y 65 años, con estudios superiores y de un nivel de ingresos medio. Viajan por ocio y vacaciones, por primera vez y en pareja, pernoctando entre 2 y 3 noches en hoteles, gastando de media poco menos de $130 €$ al día, principalmente en alojamiento, alquiler de vehículo, comida y transporte - por este orden-. El turista recomendaría el destino y, de hecho, lo ha conocido gracias a recomendaciones de amigos y familiares. Se trata de personas que se consideran alegres y abiertas, con una vida cómoda.

De este modo, la hipótesis de partida queda validada, al comprobarse que el turista tipo que visita úbeda y Baeza coincide, respecto a la mayoría de las variables sociodemográficas, en la caracterización del turista que han determinado anteriores estudios del perfil del turista de otras $\mathrm{CPH}$. Las diferencias principales se encuentran en aspectos relacionados con el grado de satisfacción del turista con determinadas facetas del destino, la planificación del viaje y las actividades del turista en destino. En materia de gasto, partiendo de la base de que el visitante de CPH gasta más que el que visita otro tipo de destino no cultural, el que acude a úbeda y Baeza realiza un mayor gasto medio diario que el que lo hace a otros destinos Patrimonio de la Humanidad.

Los resultados obtenidos son de gran importancia para la gestión del turismo en estos destinos, ya que conocer el perfil del turista que actualmente visita estas ciudades, permite encauzar los esfuerzos hacia una determinada dirección para atraer más turistas y de mayor calidad.

Como futuras líneas de investigación, sería interesante ampliar el estudio a otras CPH y comparar los resultados, además de conocer de un modo específico qué factores influyen en el gasto turístico realizado por los turistas en estas ciudades y segmentar mediante clústers los turistas según sus características y la composición del gasto que realizan. 
Declaración responsable: Las/os autoras/es declaran que no existe ningún conflicto de interés con relación a la publicación de este artículo. Las tareas se han distribuido de la siguiente manera: el artículo ha sido coordinado por J.I. Pulido. El trabajo de campo y elaboración de la base de datos han sido realizados por A.B. Mudarra. La revisión bibliográfica y redacción del marco teórico ha corrido a cargo de I. Carrillo, mientras que el análisis cuantitativo ha sido realizado por J.I. Pulido. Las/os tres autoras/es han participado en la redacción de las conclusiones y en la revisión final del texto. 


\section{Bibliografía}

Balcar, M. J., \& Pearce, D. G. (1996). Heritage tourism on the west coast of New Zealand. Tourism management, 17(3), 203-212. https://doi.org/10.1016/0261-5177(96)00007-6.

Bourdieu, P. (1986). The Forms of Capital. In J. G., Richardson, Handbook of Theory and Research for the Sociology of Education (pp. 241-59). Westport, CT: Greenwood.

Cárdenas-García, P. J., Pulido-Fernández, J. I., \& Fernández, A. B. M. (2014). Direct economic impact of tourism on World Heritage Cities: An approach to measurement in emerging destinations. Czech Journal of Tourism, 3(2), 91-106.

Correia, A., Kozak, M., \& Ferradeira, J. (2010). Impact of culture on tourist decision-making styles. International Journal of Tourism Research, 13(5), 433-446. https://doi.org/10.1002/jtr.817

Chhabra, D., Healy, R., \& Sills, E. (2003). Staged authenticity and heritage tourism. Annals of Tourism Research, 30(3), 702-719.

Craik, J. (2002). The culture of tourism. In Touring cultures (pp. 123-146). Abingdon-on-Thames: Routledge.

Gregori, P. E. M., \& Jarauta, N. G. (2015). Perfil del turista cultural en ciudades patrimoniales: los casos de San Cristóbal de La Laguna y Córdoba (España). International journal of scientific Management and Tourism, 1(2), 217-229.

ICOMOS (1976). Carta de Turismo Cultural. Comité Científico Internacional de Itinerarios Culturales (CIIC) del ICOMOS. Belgium: ICOMOS.

Instituto de Estadística y Cartografía de Andalucía (2018). Encuesta de Ocupación Hotelera. Explotación de la Encuesta de Ocupación Hotelera 2017. Sevilla: Instituto de Estadística y Cartografía de Andalucía.

Junta de Andalucía (2010). Balance del año turístico en Andalucía 2009. Sevilla: Servicio de Publicaciones de la Junta de Andalucía.

Kim, H., Cheng, C. K., \& O'Leary, J. T. (2007). Understanding participation patterns and trends in tourism cultural attractions. Tourism Management, 28(5), 1366-1371.

Kruger, M., \& Saayman, M. (2014). The determinants of visitor length of stay at the Kruger National Park. Koedoe, 56(2), Art. 1114, $11 . \quad$ Retrieved from $\quad$ http://www.scielo.org.za/scielo.php?pid=S0075$\underline{64582014000200003 \& \text { script=sci_arttext\&tlng=pt }}$ 
Lara, F., \& López Guzmán, T.J. (2004). El turismo como motor de desarrollo económico en ciudades patrimonio de la humanidad. Pasos. Revista de Turismo y Patrimonio Cultural, 2(2), 243256.

Lynch, M.F., Duinker, P. N., Sheehan, L. R., \& Chute, J. E. (2010), The demand for Mi'kmaw cultural tourism: Tourist perspectives. Tourism Management, 32(5), 977-986.

López C. (2014). Nacido en guerra. HistoriaAtocha $4^{\circ} \mathrm{C}$. La huella de la Guerra Civil en nuestras familias. Retrieved from: http://huellahistoriatocha4c.blogspot.com/

Marrero-Rodríguez, J. R., \& Abdul-Jalbar, B. (2012). Turismo Cultural y Planificación del Viaje: un Estudio de Caso. Tourism \& Management Studies, 8, 41-47. Retrieved from http://www.scielo.mec.pt/scielo.php?script=sci_arttext\&pid=S218284582012000100005\&lng=pt\&thng=es.

Morales-Fernández, E. J., \& Lanquar, R. (2014). El futuro turístico de una ciudad Patrimonio de la Humanidad: Córdoba 2031. Tourism \& Management Studies, 10(2), 7-16.

Morére, N., \& Perelló, S. (2013). Turismo cultural: patrimonio, museos y empleabilidad. Boletín Científico de las Ciencias Económico Administrativas del ICEA, 5(9). https://doi.org/10.29057/icea.v5i9.2093

Munt, I. (1994): The Other postmodern tourism: culture, travel and the new middle classes. Theory, Culture \& Society, 11(3), 101-123. https://doi.org/10.1177/026327694011003005

Poria, Y., Butler, R., \& Airey, D. (2001). Clarifying heritage tourism. Tesis doctoral. Amsterdam: Pergamon-Elsevier Science.

Poria, Y., Butler, R., \& Airey, D. (2003). The core of heritage tourism. Annals of Tourism Research, 30(1), 238-254.

Poria, Y., Butler, R., \& Airey, D. (2006). Tourist perceptions of heritage exhibits: A comparative study from Israel. Journal of Heritage Tourism, 1(1), 51-72.

Prada-Trigo, J., Chillogallo, D. A., Moreno, R. P., \& López, K. R. (2015). De los estudios económicos en turismo a la perspectiva cultural: algunas evidencias metodológicas a partir del caso de estudio de Cuenca (Ecuador). Anais Brasileiros de Estudos Turísticos-ABET, 5(1), 22-31.

Pulido-Fernández, J. I., Cárdenas-García, P. J., \& Carrillo-Hidalgo, I. (2017). Explaining tourism expenditure based on trip characteristics in emerging urban-cultural destinations. Tourism Review, 72(1), 68-86.

Pulido-Fernández, J. I., Cárdenas-García, P. J., \& Carrillo-Hidalgo, I. (2016). Trip Cultural Activities and Tourism Expenditure in Emerging Urban-cultural Destinations. International Journal of Tourism Research, 18(4), 286-296. 
Pulido-Fernández, J. I. \& Ruiz-Lanuza, A. (2017). Propuesta para una agenda de investigación de los impactos del turismo en los destinos Patrimonio de la Humanidad. Boletín de la Asociación de Geógrafos Españoles, 73, 389-411. http://dx.doi.org/10.21138/bage.2423

Richards, G. (1996). Cultural tourism in Europe. Oxfordshire: Cab International.

Richards, G. (2002). Tourism attraction systems. Exploring Cultural Behavior. Annals of Tourism Research, 29(4), 1048-1064.

Richards, G. (2018). Cultural tourism: A review of recent research and trends. Journal of Hospitality and Tourism Management, 36, 12-21.

Silberberg, T. (1995). Cultural tourism and business opportunities for museums and heritage sites. Tourism Management, 16(5), 361-365.

Su, Y. W., \& Lin, H. L. (2014). Analysis of international tourist arrivals worldwide: The role of world heritage sites. Tourism Management, 40, 46-58.

Troitiño, M. Á., \& Troitiño, L. (2009). Turismo y patrimonio en Castilla y León: las ciudades Patrimonio de la Humanidad (Ávila, Salamanca y Segovia) como destinos turísticos de referencia. Polígono. Revista de Geografía, 19, 145-178.

UNESCO (United Nations Educational, Scientific and Cultural Organization) (2009): Patrimonio Mundial de España: número especial. Paris: UNESCO, World Heritage Centre.

UNESCO (United Nations Educational, Scientific and Cultural Organization) (2014): Revista del patrimonio mundial: número especial, 73. Paris: UNESCO, World Heritage Centre.

UNESCO (United Nations Educational, Scientific and Cultural Organization) (2017): Operational Guidelines for the Implementation of the World Heritage Convention. Paris: UNESCO, World Heritage Centre.

UNWTO, World Tourism Organization (2005). City tourism \& culture the european experience. Madrid: UNWTO.

UNWTO, World Tourism Organization (2015). La Conferencia Mundial sobre Turismo y Cultura de la OMT y la UNESCO reúne por primera vez a ministros de Turismo y de Cultura (Press Release no: 15012). Madrid: UNWTO.

UNWTO, World Tourism Organization (2018). UNWTO Tourism Highlights, 2018 Edition. Madrid: UNWTO. https://doi.org/10.18111/9789284419876.

Van der Borg, J., Costa, P., \& Gotti, G. (1996). Tourism in European heritage cities. Annals of Tourism Research, 23(2), 306-321. 
Van Der Ark, L. A., \& Richards, G. (2006), Attractiveness of the cultural activities in European cities: a latent class approach. Tourism Management, 27, 1408-1413.

Zeppel, H., \& Hall, M. (1991). Selling art and history: Cultural heritage and tourism. Journal of Tourism Studies, 2(1), 29-45. 